\title{
IDADE E CRESCIMENTO DE LOPHOLATILUS VILLARII RIBEIRO NO SUDESTE DO BRASIL (OSTEICHTHYES, MALACANTHIDAE)
}

\author{
Maria Odete Ximenes Carvalho ${ }^{1}$ \\ Rafael de Almeida Tubino ${ }^{2}$ \\ Melquíades Pinto Paiva ${ }^{2,3}$ \\ Magda Fernandes de Andrade-Tubino ${ }^{2}$ \\ Antonio Adauto Fonteles-Filho ${ }^{1,3}$
}

\begin{abstract}
AgE AND GROWTH OF LOPHOLATILUS VILLARI RiBEIRO AT SOUTHEAST OF BRAZIL (Osteichthyes, MalacANTHIDAE). The tilefish, Lopholatilus villarii Ribeiro, 1915 is a commercially-important demersal fish, in the Southeast Brazil, inhabiting calcareous and rocky substrates of the outer shelf and upper continental slope. This study was based on readings of the scale's age rings of 153 especimens, caught from March, 1995 to March, 1996. Scales were found to grow on an isometric proportion with fish length, and age rings are formed between the third and fourth trimesters (winter and spring). The age groups of sampled fish varied from II to X years, and the growth equation in total length $(\mathrm{cm})$ is $\mathrm{L}_{\mathrm{t}}=113.4\left[1-\mathrm{e}^{-0.144(\mathrm{t}+1.4)}\right]$ with the correspondent one in weight $\left.(\mathrm{kg}): \mathrm{W}_{\mathrm{t}}=22.5\left[1-\mathrm{e}^{-0.144}(\mathrm{t}+1.4)\right]\right]^{3.184}$ obtained from the weight/length regression equation: $\ln \mathrm{W}=-11.95+3.184 \ln \mathrm{L}$. The tilefish has a low growth coefficient $(K=0.144)$, typical of carnivorous species of the fourth trophic level, and it may reach, theoretically, 41 years of age.

KEY WORDS. Lopholatilus villarii, age and growth, Brazilian ichthyofauna
\end{abstract}

O batata, Lopholatilus villarii Ribeiro, 1915, é uma espécie da família Malacanthidae (NELSON 1994), com distribuição geográfica no Atlântico Ocidental, desde o arquipélago de Abrolhos (Brasil) até o norte da Argentina (Dooley 1978; FigUEIREDO \& MENEZES 1980; PAIVA 1997). É encontrado em fundos de cascalho e rocha, nas partes externa da plataforma e superior do talude continentais, sendo capturado em pesqueiros com até $650 \mathrm{~m}$ de profundidade. Alimenta-se de peixes, crustáceos e equinodermos (CARVALHO-FILHO 1992).

Este peixe tem grande importância econômica na região sudeste do Brasil, tendo participado com $32,4 \%$ do total desembarcado pela frota de barcos linheiros nos portos do Rio de Janeiro/Niterói e Vitória, nos anos de 1986-1995.

Existem poucas informações publicadas sobre a biologia pesqueira e dinâmica populacional do batata (PAIVA 1997), mas elas não tratam da idade e crescimento da espécie, aspectos que constituem os objetivos deste trabalho.

1) Laboratório de Ciências do Mar, Universidade Federal do Ceará. Avenida da Abolição 3207 , 60165-081 Fortaleza, Ceará, Brasil.

2) Departamento de Biologia Marinha, Universidade Federal do Rio de Janeiro. Cidade Universitária, 21949-900 Rio de Janeiro, Rio de Janeiro, Brasil.

3) Bolsista do CNPq.

Revta bras. Zool. 15 (4): 889 - 906, 1998 


\section{MATERIAL E MÉTODOS}

As amostragens foram realizadas no período de março/1995 a março/1996, nos desembarques da frota de linheiros em Niterói, com a coleta de escamas de 228 indivíduos, com registro do comprimento total (em centímetros) e peso total (em quilogramas). A análise foi feita para machos e fêmeas em conjunto, por impossibilidade de identificação dos sexos, porque os peixes são comercializados sob a forma inteira (não-eviscerada) e não apresentam caracteres sexuais externos.

Foram retiradas escamas da região axial da nadadeira peitoral, onde elas são simétricas, com baixo percentual de regeneração e espessura menor do que naquelas encontradas em outras áreas do corpo. Do total de indivíduos amostrados, foram selecionados 153 (67,1\% de aproveitamento), cujas escamas apresentavam foco com nitidez suficiente para a correta leitura e contagem dos anéis etários.

A identificação destes foi realizada em tela de um Projetor Eberbach, com aumento de 40X, com medições entre o foco e cada um dos anéis, e também entre o foco e a borda da escama. De cada peixe foram selecionadas três escamas e feitas duas leituras dos seus anéis etários.

A existência de proporcionalidade entre o crescimento do peixe e o da escama foi determinada ajustando-se uma regressão linear aos dados pela equação funcional $\mathbf{Y}=\mathbf{a}+\mathbf{b X}$, para um nível de significância $\alpha=0,01$.

A periodicidade no processo de formação dos anéis de crescimento foi analisada através da variação mensal do incremento marginal (IM), definido pela fórmula:

$$
I M=\frac{R-r_{n}}{R-r_{n-1}} \rightarrow(1)
$$

onde $\mathbf{R}$ é a distância do foco à borda anterior da escama, e $\mathbf{r}_{\mathbf{n}}$ e $\mathbf{r}_{\mathbf{n}}-\mathbf{1}$ são, respectivamente, as distâncias do foco ao último e penúltimo anéis etários, segundo CALDWALLADER (1978). Deve-se ressaltar que o valor de IM não foi calculado para o mês de setembro de 1995, por falta de dados.

O comprimento total em cada idade foi retrocalculado segundo a fórmula:

$$
L^{\prime}=\frac{S^{\prime}(L-a)}{S}+a \rightarrow(2)
$$

onde $\mathbf{L}$ e $\mathbf{S}$ correspondem aos comprimentos do peixe e da escama na captura, $\mathbf{L}$ ' e S' correspondem aos comprimentos do peixe e da escama nas diversas idades, e a é o coeficiente linear da regressão entre $\mathbf{L}$ e $\mathbf{S}$ (FonTELES-FILHO 1989).

Para correlacionar o comprimento $(\mathrm{cm})$ com a idade (ano), ajustamos aos dados à equação de BERTALANFFY (1938), em comprimento total (cm):

$$
L_{t}=L_{\infty}\left[1-e^{-K(t-t o)}\right] \rightarrow(3)
$$

e peso $(\mathrm{kg})$ :

$$
W_{t}=W_{\infty}\left[1-e^{-K(t-t o)]^{b}} \rightarrow(4)\right.
$$

onde $\mathbf{L}_{\mathbf{t}} \mathrm{e} \mathbf{W}_{\mathbf{t}}$ são o comprimento e o peso na idade $\mathbf{t} ; \mathbf{L}_{\infty} \mathrm{e} \mathbf{W}_{\infty}$ são o comprimento 
e o peso máximos teóricos; $\mathbf{K}$ é o coeficiente de crescimento; $\mathbf{t}_{\mathbf{o}}$ é a idade teórica em que o indivíduo teria comprimento zero se tiver crescimento de acordo com a equação 3; e b é o coeficiente angular da regressão peso/comprimento.

A obtenção da curva de crescimento em peso foi feita através da relação peso/comprimento, determinada pela medição de 226 indivíduos de ambos os sexos, conforme a equação de regressão:

$$
\ln W=\ln A+b \ln L \rightarrow
$$

Considerando-se $\Delta t=1$ ano, a relação Ford-Walford, que correlaciona os comprimentos do indivíduo em grupos-de-idade sucessivos $t$ e $t+1$, pode ser descrita pela equação:

$$
L_{t+1}=L_{\infty}\left(1-e^{-K}\right)+e^{-K} L_{t} \rightarrow(6)
$$

No ponto em que uma bissetriz intercepta a reta de regressão, registra-se a igualdade $\mathbf{L}_{\mathbf{t}}=\mathbf{L}_{\mathbf{t}+\mathbf{1}}=\mathbf{L}_{\infty}$, já que a taxa de crescimento de um peixe que atingiu seu tamanho máximo teórico é igual a zero. Desse modo, substituindo-se $\mathbf{L}_{\mathbf{t}} \mathbf{e} \mathbf{L}_{\mathbf{t}+\mathbf{1}}$ por $\mathbf{L}_{\infty}$, a Equação 6 passa a ser representada simplesmente pela reta de regressão $\mathbf{L}_{\infty}$ $=\mathbf{a}+\mathbf{b} \mathbf{L}_{\infty}$, a partir da qual se estima o valor de $\mathbf{L}_{\infty}$ :

$$
L_{\infty}=\frac{a}{1-b} \rightarrow(7)
$$

A partir da Equação 6, em que $\mathrm{e}^{-\mathrm{K}}=\mathrm{b}$, por logaritmização de ambos os termos, tem-se:

$$
K=-\ln b \rightarrow(8)
$$

$\mathrm{O}$ valor de $\mathbf{t}_{\mathbf{0}}$, deduzido da Equação 3, foi calculado pela fórmula:

$$
t_{o}=t+\frac{1}{K} \ln \left(\frac{L_{\infty}-L_{t}}{L_{\infty}}\right) \rightarrow(9)
$$

\section{RESULTADOS E DISCUSSÃO}

Os valores retrocalculados correspondentes ao comprimento total de $L$. villarii, quando da formação de cada anel, encontram-se na tabela I, com registro de indivíduos nos grupos-de-idade de II $(38,5 \mathrm{~cm})$ a X anos $(90,0 \mathrm{~cm})$.

As escamas do batata são do tipo ctenóide, de fina espessura, com os anéis de crescimento evidenciados pela diminuição das distâncias entre os circuli na parte anterior da escama. Em alguns casos, é possível a observação destes anéis também no campo posterior da escama, onde aparecem como interrupções nas fileiras dos ctênios (Fig. 1).

A dependência do comprimento total (L), na faixa de 32,6 -90,0 cm em relação ao comprimento da escama $(\mathbf{S})$, na faixa de $0,44-0,95 \mathrm{~cm}$, foi determinada através da regressão linear, expressa pela equação: $\mathrm{L}=-10,2+106,0 \mathrm{~S}(\mathrm{r}=0,902$; $\mathrm{P}<0,01)$.

A elevada correlação estatística entre $\mathbf{L}$ e $\mathbf{S}$ satisfaz a premissa de isometria na relação entre os comprimentos do peixe e da escama. No entanto, a obtenção de um elevado valor negativo do coeficiente linear $(\mathbf{a}=-10,2 \mathrm{~cm})$ da regressão entre 
os comprimentos do peixe e da escama, significa que a razão $\mathbf{L} / \mathbf{S}$ tende a aumentar com o tamanho individual, ou seja, a escama é relativamente menor em peixes menores. Portanto, segundo o fenômeno descrito por LEE (1920), indivíduos pertencentes às coortes mais velhas aparentemente eram menores quando se encontravam no grupo-de-idade I, do que aqueles das coortes mais jovens.

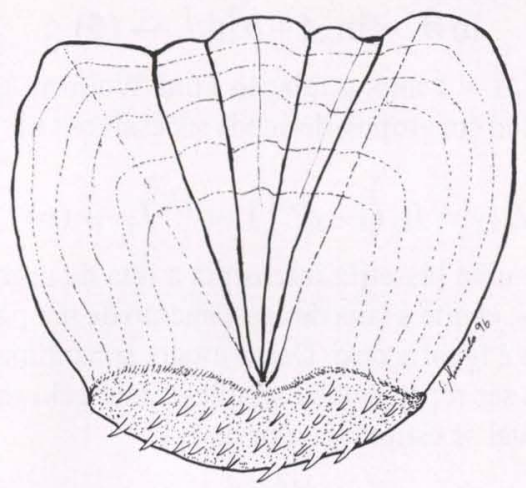

Fig. 1. Escama de um exemplar de Lopholatilus villarii, com $54,5 \mathrm{~cm}$ de comprimento total e cinco anéis etários.

Tabela I. Valores retrocalculados do comprimento total de Lopholatilus villarii, por grupos-deidade, na região sudeste do Brasil.

\begin{tabular}{|c|c|c|c|c|c|c|c|c|c|c|c|c|}
\hline \multirow{2}{*}{$\begin{array}{c}\text { Grupo de } \\
\text { idade (ano) }\end{array}$} & \multirow{2}{*}{$\begin{array}{c}\text { Comprimento } \\
\text { na captura }(\mathrm{cm})\end{array}$} & \multirow{2}{*}{$\begin{array}{l}\text { Número de } \\
\text { observaçōes }\end{array}$} & \multicolumn{10}{|c|}{ Comprimento retrocalculado $(\mathrm{cm})$ por grupo de idade } \\
\hline & & & 1 & ॥ & III & IV & V & VI & VII & VIII & IX & $\mathrm{x}$ \\
\hline II & 38,5 & 7 & 20,7 & 33,5 & & & & & & & & \\
\hline III & 42,2 & 15 & 20,2 & 32,6 & 40,1 & & & & & & & \\
\hline IV & 53,2 & 45 & 20,6 & 34,1 & 44,0 & 51,0 & & & & & & \\
\hline v & 59,4 & 37 & 19,9 & 33,6 & 44,3 & 52,0 & 57,7 & & & & & \\
\hline VI & 66,6 & 25 & 21,2 & 34,2 & 44,9 & 53,1 & 59,6 & 64,6 & & & & \\
\hline VII & 75,2 & 15 & 20,9 & 34,7 & 45,7 & 55,3 & 63,2 & 69,1 & 72,9 & & & \\
\hline VIII & 82,8 & 6 & 19,6 & 33,0 & 43,6 & 54,0 & 63,1 & 70,3 & 76,2 & 80,8 & & \\
\hline IX & 85,0 & 2 & 22,5 & 35,7 & 47,0 & 57,1 & 64,7 & 72,3 & 77,4 & 81,1 & 83,7 & \\
\hline$x$ & 90,0 & 1 & 16,2 & 29,4 & 39,9 & 49,1 & 58,4 & 66,3 & 74,2 & 80,8 & 86,0 & 87,9 \\
\hline \multicolumn{3}{|c|}{ Comprimento méio $(\mathrm{cm})$} & 20,5 & 33,8 & 44,0 & 52,4 & 59,8 & 67,0 & 74,1 & 80,9 & 84,5 & 87,9 \\
\hline \multicolumn{3}{|c|}{ Número de indivíduos } & 153 & 153 & 146 & 131 & 86 & 49 & 24 & 9 & 3 & 1 \\
\hline
\end{tabular}

Para se avaliar a influência desse vício sobre a estimação dos parâmetros da curva de crescimento do batata, os comprimentos retrocalculados, a partir de escamas com 2 a 10 anéis etários, foram plotados em função da amplitude de valores observados para cada grupo-de-idade (Fig. 2). Pode-se verificar que, para os grupos-de-idade I e II, existe uma tendência para estabilização dos valores ao longo da amplitude de comprimento, mas estes passam a assumir uma tendência crescente a partir do grupo-de-idade III. Tais resultados indicam a ocorrência do fenômeno de Lee em parte da estrutura etária, o que poderia interferir nos valores do comprimento retrocalculado. No entanto, a introdução do valor de $\mathbf{a}=-10,2$ na fórmula original do retrocálculo (equação 2) funciona como fator de correção e minimiza a 
influência desse vício sobre os valores retrocalculados; desse modo, pode-se considerar que a curva de crescimento ajustada a partir dos dados reflete, com razoável grau de precisão, o padrão de desenvolvimento corporal dos indivíduos de L. villarii .
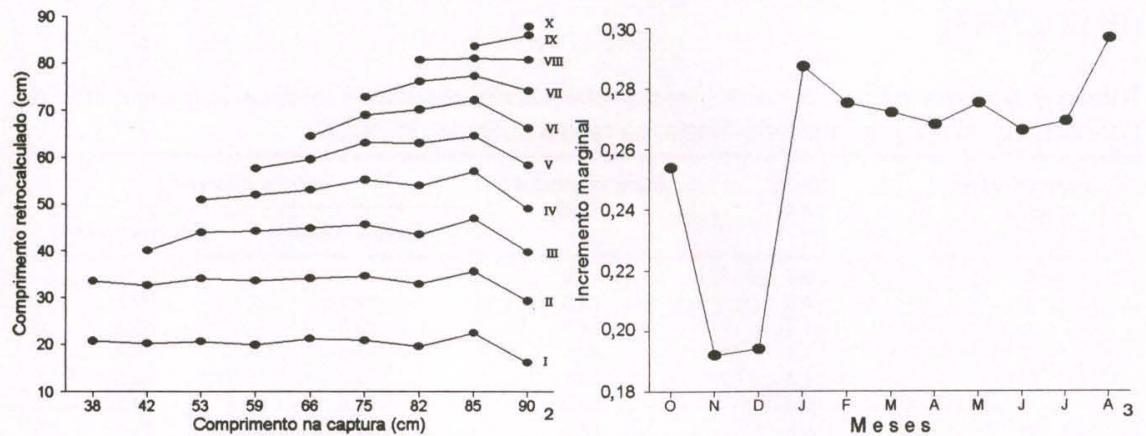

Figs 2-3. Lopholatilus villarii. (2) Variação do comprimento médio, por grupo-de-idade, retrocalculado em função do comprimento na captura; (3) Variação mensal do incremento marginal em anéis etários das escamas.

Quanto à periodicidade anual na formação do anel, a variação mensal do incremento marginal (Fig. 3) indica, inicialmente, uma tendência de decréscimo desse parâmetro nos meses de outubro-dezembro, após um período de valores elevados que se estende de janeiro a agosto. A redução da largura do anel etário em novembro-dezembro (com valores de IM iguais a 0,192 e 0,194 ) e o rápido aumento em janeiro (IM $=0,287)$, sugerem que a mudança de idade ocorre entre o terceiro e quarto trimestres do ano, indicando o início da formação de um novo anel etário, ou seja, o indivíduo passou recentemente a integrar o grupo-de-idade seguinte na população. A escassez de informações sobre a biologia da espécie dificulta identificar qual seria o fator causal responsável pela periodicidade, mas geralmente esta se relaciona com a reprodução em espécies da zona tropical, ou variações estacionais marcantes na temperatura, como ocorre com espécies da zona temperada.

A regressão entre valores do comprimento em grupos-de-idade anuais sucessivos é expressa pela seguinte equação: $\mathrm{L}_{t+1}=15,20+0,866 \mathrm{~L}_{t}(\mathrm{r}=0,999 ; \mathrm{P}<$ 0,01 ), cujos coeficientes $\mathbf{a}$ e $\mathbf{b}$ foram introduzidos nas Equações 7 e 8 para a estimação de $\mathbf{L}_{\infty}=113,4 \mathrm{~cm}$ e $\mathbf{K}=0,144$, juntamente com o valor de $\mathbf{t}_{\mathbf{0}}=-1,4$ ano (equação 9) no ajuste da seguinte equação de crescimento em comprimento total (cm): $\mathrm{L}_{\mathrm{t}}=113,4\left[1-\mathrm{e}^{-0,144(\mathrm{t}+1,4)}\right]$.

Atribuindo-se valores médios a $\mathbf{t}$ nos grupos-de-idade $0(0,5$ ano) a XXX (30,5 anos), foram determinados os respectivos comprimentos individuais, e as taxas absoluta e relativa de crescimento (Tab. II; Fig. 4). O padrão de crescimento mostra 
que o batata cresce rapidamente de tamanho nos primeiros grupos-de-idade, correspondentes ao estoque jovem; em seguida, são observadas taxas de crescimento gradativamente decrescentes, tendendo para uma estabilização a partir de 18 anos de idade, quando não acrescenta mais do que $1,2 \%$ do tamanho anterior entre dois grupos-de-idade consecutivos. O batata atinge uma idade teórica máxima de 41 anos, resultante de um valor de $\mathbf{L}_{\infty}=113,4 \mathrm{em} \mathbf{C T}$, e apresenta baixo coeficiente de crescimento $(\mathbf{K}=0,144)$, característico de espécies carnívoras de terceiro grau (PAUly 1983).

Tabela II. Valores médios do comprimento total e taxas absoluta e relativa de crescimento de Lopholatilus villarii, por grupo-de-idade, na região sudeste do Brasil.

\begin{tabular}{|c|c|c|c|c|}
\hline \multirow{2}{*}{$\begin{array}{l}\text { Grupo de idade } \\
\text { (ano) }\end{array}$} & \multirow{2}{*}{$\begin{array}{l}\text { Idade } \\
\text { (ano) }\end{array}$} & \multirow{2}{*}{$\begin{array}{l}\text { Comprimento médio } \\
(\mathrm{cm})\end{array}$} & \multicolumn{2}{|c|}{ Taxa de crescimento } \\
\hline & & & Absoluta (cm/ano) & Relativa (\%/ano) \\
\hline 0 & 0,5 & 7,9 & - & - \\
\hline 1 & 1,5 & 22,0 & 14,1 & 178,5 \\
\hline ॥ & 2,5 & 34,3 & 12,3 & 55,9 \\
\hline III & 3,5 & 44,9 & 10,6 & 30,9 \\
\hline IV & 4,5 & 54,1 & 9,2 & 20,5 \\
\hline v & 5,5 & 62,0 & 7,9 & 14,6 \\
\hline VI & 6,5 & 68,9 & 6,9 & 11,1 \\
\hline VII & 7,5 & 74,9 & 6,0 & 8,7 \\
\hline VIII & 8,5 & 80,1 & 5,2 & 6,9 \\
\hline IX & 9,5 & 84,5 & 4,4 & 5,5 \\
\hline$x$ & 10,5 & 88,4 & 3,9 & 4,6 \\
\hline $\mathrm{XI}$ & 11.5 & 91,8 & 3,4 & 3,8 \\
\hline XII & 12,5 & 94,7 & 2,9 & 3,2 \\
\hline XIII & 13,5 & 97,2 & 2,5 & 2,6 \\
\hline XIV & 14,5 & 99,3 & 2,1 & 2,2 \\
\hline$X V$ & 15,5 & 101,2 & 1,9 & 1,9 \\
\hline $\mathrm{XVI}$ & 16,5 & 102,9 & 1,7 & 1,7 \\
\hline XVII & 17,5 & 104,3 & 1,4 & 1,4 \\
\hline XVIII & 18,5 & 105,5 & 1,2 & 1,2 \\
\hline XIX & 19,5 & 106,5 & 1,1 & 1,0 \\
\hline$x x$ & 20,5 & 107,5 & 0,9 & 0,8 \\
\hline$\hat{X X I}$ & 21,5 & 108,3 & 0,8 & 0,7 \\
\hline XXII & 22,5 & 109,0 & 0,7 & 0,6 \\
\hline XXIII & 23,5 & 109,6 & 0,6 & 0,6 \\
\hline XXIV & 24,5 & 110,1 & 0,5 & 0,5 \\
\hline$X X V$ & 25,5 & 110,5 & 0,4 & 0,4 \\
\hline XXVI & 26,5 & 110,9 & 0,4 & 0,4 \\
\hline XXVII & 27,5 & 111,2 & 0,3 & 0,3 \\
\hline XXVIII & 28,5 & 111,5 & 0,3 & 0,3 \\
\hline$X X I X$ & 29,5 & 111,8 & 0,3 & 0,3 \\
\hline$X X X$ & 30,5 & 112,0 & 0,2 & 0,2 \\
\hline
\end{tabular}

Por transformação do comprimento em peso, através da equação de regressão calculada com base nos dados da tabela III: $\ln \mathrm{W}=-11,95+3,184 \ln \mathrm{L}(\mathrm{r}=0,991$; $\mathrm{P}<0,01)$, foi obtida a seguinte equação de crescimento em peso $(\mathrm{kg}): \mathrm{W}_{\mathrm{t}}=22,5$ $\left[1-\mathrm{e}^{-0,1444(\mathrm{t}+1,4)}\right]^{3,184}$

O gênero Lopholatilus Goode \& Bean, 1880 abriga apenas duas espécies, ambas ocorrendo ao longo das costas atlânticas das Américas: a espécie-tipo é $L$. chamaeleonticeps, encontrada desde a Nova Scotia (Canadá) até o Suriname (DoOLEY 1978); a outra espécie é a estudada neste trabalho.

Para a espécie $L$. chamaeleonticeps, existem informações sobre dois dos seus estoques, em águas dos Estados Unidos da América, relativas a parâmetros de 
crescimento: na região de New England (águas temperadas), TURNER et al. (1983) encontraram os valores de $\mathbf{K}=0,162$ e $\mathbf{L}_{\infty}=97,6 \mathrm{~cm} \mathbf{C Z}(=100,5 \mathrm{~cm} \mathbf{C T})$; nas águas subtropicais da Georgia os valores destes parâmetros foram $\mathbf{K}=0,084$ e $\mathbf{L}_{\infty}$ $=90,7 \mathrm{~cm} \mathrm{CZ}$ (=93,4 cm CT), segundo HARRIS \& GROSSMAN (1985).

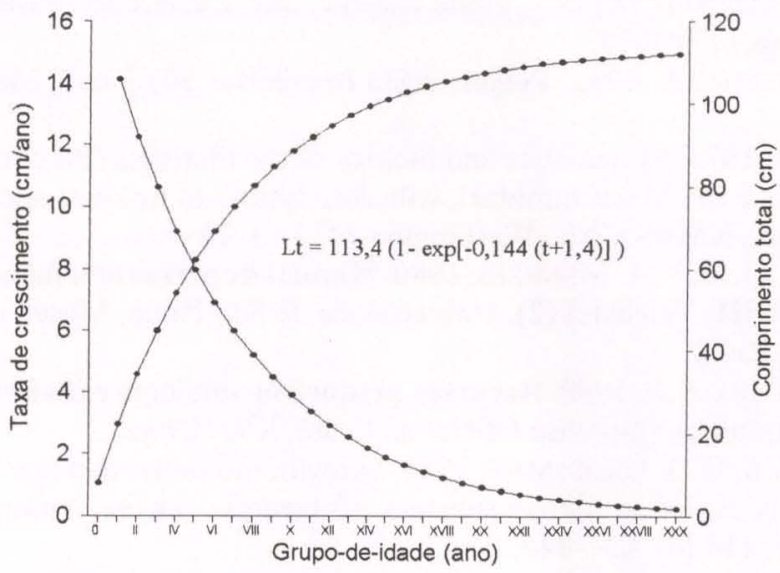

Fig. 4. Curvas de crescimento absoluto e relativo, em comprimento, de Lopholatilus villarii no periodo de março/1995 a março/1996, na região sudeste do Brasil.

Tabela III. Dados básicos de comprimento e peso, utilizados no ajuste da equação de regressão peso/comprimento de Lopholatilus villarii, na região sudeste do Brasil.

\begin{tabular}{cccc}
\hline \multirow{2}{*}{$\begin{array}{c}\text { Comprimento total } \\
(\mathrm{cm})\end{array}$} & Frequéncia & \multicolumn{2}{c}{ Peso total $(\mathrm{Kg})$} \\
\cline { 3 - 4 } & & Observado & Calculado \\
\hline 32,5 & 3 & 0,467 & 0,421 \\
37,5 & 7 & 0,767 & 0,664 \\
42,5 & 15 & 1,033 & 0,988 \\
47,5 & 15 & 1,460 & 1,409 \\
52,5 & 34 & 1,900 & 1,937 \\
57,5 & 35 & 2,554 & 2,588 \\
62,5 & 31 & 3,345 & 3,375 \\
67,5 & 23 & 4,161 & 4,312 \\
72,5 & 19 & 5,511 & 5,414 \\
77,5 & 12 & 7,033 & 6,694 \\
82,5 & 16 & 8,188 & 8,169 \\
87,5 & 8 & 10,238 & 9,852 \\
92,5 & 5 & 11,780 & 11,759 \\
97,5 & 3 & 13,667 & 13,905 \\
\hline
\end{tabular}

AGRADECIMENTOS. Somos gratos ao Sr. João Evangelista Nunes de Viveiros e seus empregados, pelas facilidades que nos proporcionaram em Niterói, para a condução das amostragens e coleta de material que suportam o presente trabalho. Os autores Antonio Adauto Fonteles-Filho e Melquíades Pinto Paiva expressam os seus agradecimentos ao Conselho Nacional de Desenvolvimento Cientifico e Tecnológico ( $\mathrm{CNPq}$ ) pelas bolsas de pesquisa que thes foram concedidas. 


\section{REFERÊNCIAS BIBLIOGRÁFICAS}

BerTALANFFy, L.v. 1938. A quantitative theory of organic growth. (Inquiries on growth laws, II). Hum. Biol., Baltimore, 10: 181-213.

Caldwallader, P.L. 1978. Age, growth and condition of the common river galaxias, Galaxias vulgaris, in the Glentui river, Canterbury. Fish. Res. Bull., Wellington, (17): 9-35.

Carvalho-Filho, A. 1992. Peixes: costa brasileira. São Paulo, Marca d'Água, $304 p$.

DooLEY, J.K. 1978. Systematics and biology of the tilefishes (Perciformes: Branchiostegidae and Malacanthidae), with descriptions of two new species. NOAA Tech. Rep., NMFS Circ., Washington, (411): 1-78.

FigueirEDo, J.L. \& N.A. MENEZES. 1980. Manual de peixes marinhos do sudeste do Brasil. III. Teleostei (2). Universidade de São Paulo, Museu de Zoologia, 90p., São Paulo.

FONTELES-FILHO, A.A. 1989. Recursos pesqueiros: biologia e dinâmica populacional. Fortaleza, Imprensa Oficial do Ceará, XVI+296p.

HARRIS, M.J. \& G.D. GROSSMAN. 1985. Growth, mortality, and age composition of a lightly exploited tilefish substock off Georgia. Trans. Amer. Fish. Soc., Lawrence, 114 (6): 837-846.

LEE, R. 1920. A review of the methods of age and growth determination in fishes by means of scales. Fish. Invest., ser. 2, London, (4): 1-32.

NeLSON, J.S. 1994. Fishes of the world. New York, John Wiley \& Sons, Inc., $3^{\text {rd }}$ ed., XVII+600p.

PAIVA, M.P. 1997. Recursos pesqueiros estuarinos e marinhos do Brasil. Fortaleza, UFC Edições, 286p.

PAULY, D. 1983. On the interrelationships between natural mortality, growth parameters, and environmental temperature in 175 fish stocks. Jour. Cons. Perm. Int. Explor. Mer, Copenhagen, 39 (2): 175-192.

TURNER, S.C.; C.B. GRIMES \& K.W. ABLE. 1983. Growth, mortality and age/size structure of the fisheries for tilefish, Lopholatilus chamaelonticeps, in the middle Atlantic-southern New England region. Fish. Bull., Seattle, 81 (4): 751-763.

Recebido em 05.VI.1997; aceito em 03.XI.1998. 